\title{
Review
}

\section{Current studies on human papillomavirus in Saudi Arabia}

\author{
Fatimah S Alhamlan ${ }^{1,3}$, Ahmed A Al-Qahtani ${ }^{1,2,3}$, Mohammed N Al-Ahdal'1,3,4 \\ ${ }^{1}$ Department of Infection and Immunity, King Faisal Specialist Hospital and Research Center, Riyadh, Saudi Arabia \\ 2 Liver Disease Research Center, King Saud University, Riyadh, Saudi Arabia \\ ${ }^{3}$ Department of Microbiology and Immunology, College of Medicine, Alfaisal University, Riyadh, Saudi Arabia \\ ${ }^{4}$ Department of Pathology and Laboratory Medicine, King Faisal Specialist Hospital and Research Center, Riyadh, \\ Saudi Arabia
}

\begin{abstract}
Human papillomavirus (HPV) infection is a significant etiological factor and an important prognosticator in cervical cancer. Indeed, researchers worldwide have confirmed these roles for high-risk HVPs in over 70\% of cervical cancer cases. According to the World Health Organization, approximately 561,200 new cancer cases (5.2\% of all new cancers) are attributed to HPV infection. Over 120 types of HPV are classified further as either low-risk HPV (LR-HPV) or high-risk HPV (HR-HPV) based on their oncological potential of transforming cells. The LRHPV types cause benign hyperproliferative lesions (i.e. genital warts) while the HR-HPV types are strongly associated with premalignant and malignant cervical lesions. Data on the prevalence of HPV, survival of infected patients, and mortality rate are scarce in Saudi Arabia. The unsubstantiated assumption of a low prevalence of HPV in Saudi Arabia has contributed to limiting HPV research in this conservative country. Therefore, the goal of this review is to shed light on the current HPV research being conducted and the prevalence of HPV in Saudi Arabia.
\end{abstract}

Key words: Human Papillomavirus (HPV); Cervical Cancer; Saudi Arabia.

J Infect Dev Ctries 2015; 9(6):571-576. doi:10.3855/jidc.6538

(Received 05 January 2015 - Accepted 20 May 2015)

Copyright $(2015$ Alhamlan et al. This is an open-access article distributed under the Creative Commons Attribution License, which permits unrestricted use, distribution, and reproduction in any medium, provided the original work is properly cited.

\section{Introduction}

Cervical cancer is both the fourth most common cause of cancer and the fourth most common cause of death from cancer in women worldwide [1]. In Saudi Arabia, cervical cancer ranks as the eleventh most frequent cancer in women, and the eighth most frequent cancer among women between the ages of 15 and 44 [2]. The World Health Organization (WHO) has reported that 6.51 million women in Saudi Arabia aged 15 years and older are at risk of developing cervical cancer. Current estimates indicate that 152 women in Saudi Arabia are diagnosed with cervical cancer and 55 die from the disease each year [3]. In recognition of the global prevalence, comprehensive research efforts are ongoing to fight cancer and make this disease curable.

Human, papillomaviruses are members of a family of small, non-enveloped papillomaviruses having a double-stranded DNA (dsDNA) genome. Composed of approximately 7.9 kilobases (kb) [4], all HPVs infect either the cutaneous or mucosal surfaces of epithelial cells. Approximately 189 HPV genotypes from this diverse group of viruses have been sequenced and classified according to their phylogenetic position, biological niche, and oncogenic potential with new types discovered regularly $[5,6]$. Based on their oncogenic potential, the 30-40 types from the $\alpha$-genus of HPVs that infect the human genital tract can be subdivided into low- and high-risk types. Low-risk HPV types include HPV6 and 11. These low-risk viruses have been associated with benign anogenital warts or condylomata. By contrast, at least 12 high-risk HPV (HRHPV) types, HPV16, -18, -31, -33, -35, -39, $45,-51,-52,-56,-58$ and -59 , have been associated with anogenital cancers as well as precursor neoplastic lesions [7-9].

Nearly a decade after the German researcher Harald zur Hausen hypothesized in 1974 that cervical cancer was caused by a chronic HPV infection of the cervix, he isolated HPV 16 and a year later HPV 18 in cervical cancer cells. Although the role of HPV in cervical cancer was not firmly established using large-scale epidemiological studies and molecular techniques until 1990, Dr. Hausen shared the Nobel Prize in Physiology or Medicine in 2008 for his discovery $[10,11]$.

Human papillomavirus (HPV) infection has been established as a significant etiological factor in cervical cancer [12]. Indeed, the presence of HPV has become increasingly important as an indicator of atypical 
cervical cells and is now being used as a primary screen for cervical cancer in many clinics. Although marked progress has been made in understanding the molecular mechanisms that cause the transformation of a normal cell into a cancer cell, our knowledge is far from complete and much remains to be discovered. To date, researchers have confirmed that certain genotypes of HPV called high-risk types play a role in the development of cervical cancer; however, additional investigations are needed to gain a better understanding of HPV infection and its role in cervical carcinogenicity worldwide, particularly in Saudi Arabia. This review seeks to shed light on the HPV research currently being conducted and the prevalence of HPV in Saudi Arabia.

\section{Human papillomavirus and cervical cancer}

HPVs contain a double-stranded, closed circular DNA genome, which comprises approximately eight thousand base pairs with at least eight open reading frames that divide the genome into three regions. The first region, the long control region, includes the regulatory functions for the transcription of the E6 and E7 genes. The second region, the early region (E), contains six structural proteins, namely, E1, E2, E4, E5, $\mathrm{E} 6$, and E7, which are involved in viral replication and oncogenesis. The third region, the late region $(\mathrm{L})$, encodes L1 and L2 structural proteins that form the major and minor capsid proteins, respectively [13]. Like all papillomaviruses, HPVs establish productive infections only in the keratinocytes of the skin or mucous membranes. While the majority of the known types of HPV cause no symptoms in most people, some cause warts (verrucae), whereas others can lead to cancers of the cervix, vulva, vagina, penis, oropharynx, and anus [14]. Over 120 subtypes of HPVs are classified as either low-risk (LR-HPV) or high-risk (HR-HPV) based on their oncological potential for transforming cells. The LR-HPV types cause benign hyperproliferative lesions, or genital warts, while the HR-HPV types are strongly associated with premalignant and malignant cervical lesions [15]. An HPV type is defined as an HPV isolate with an L1 gene sequence that is at least $10 \%$ unique when compared with any other type. With advances in molecular technology (especially sequencing), more information is being revealed about HPV types, subtypes, prevalence, and risk. Recently, for example, it was discovered that type 55 and 64 share over $93 \%$ sequence homology with HPV-44 and HPV-34, respectively; thus, type 55 and 64 are no longer considered separate types. In addition, new HPV subtypes, such as HPV$68 \mathrm{a}$ and HPV-68b, were recently identified $[16,17]$.
To date, the late gene (L1) region has been the most commonly used target for HPV detection assays. Indeed, the L1 region sequences of different HPV types are sufficiently conserved to be utilized for primer design [9]. However, numerous studies have shown that the $\mathrm{L} 1$ region is a reliable target only if the virus is present in its episomal form and not in its integrated form. Because the L1 region is lost during HPV integration into the human genome, relying on L1 primers could be misleading; thus, undetected viruses could later spread and lead to disease progression [18, 19].

Whereas the L1 gene has been central for HPV detection and typing, the HPV E6 and E7 early genes are the most important viral oncoproteins perturbing the cell cycle. The E6 and E7 genes in high-risk HPVs are oncoproteins that can alter the function of cell cycle regulators. These two primary oncoproteins inactivate two tumor suppressor proteins, p53 (inactivated by E6) and $\mathrm{pRb}$ (inactivated by E7), leading to cell cycle disruption [20, 21]. In addition, these viral oncogenes are thought to modify the cell cycle so as to retain the host's differentiating keratinocytes in a state favorable for the amplification and replication of the viral genome and consequent late gene expression. Importantly, E6 and E7 genes are present in the episomal and in the integrated states of the virus and are retained in the cervical tumor. Therefore, these genes are strong candidates for use in HPV detection and viral load quantification, and have become the newly proposed targets for HPV detection in cervical cancer.

Cervical cancer is a rare complication following infection with high-risk HPV, but every abnormal or dysplastic lesion of the cervix is potentially malignant and over time may develop into cervical cancer. The development of cervical cancer is considered a multistep process in which HPV is necessary, but it alone is an insufficient cause [22-24]. Disease develops only in the presence of a persistent cervical epithelium HPV infection. Abnormal cervical epithelial cells can be detected microscopically with Pap staining, and most laboratories worldwide perform the Pap smear test as a primary screen of cervical tissue, reporting the results according to the Bethesda System. Based on this system, squamous cell abnormalities are divided into the following categories, ranging from the mildest to the most severe results: atypical squamous cells of undetermined significance (ASCUS), low-grade squamous intraepithelial lesions (LGSIL), high-grade squamous intraepithelial lesions (HGSIL), and squamous cell carcinoma [25]. This forms the basis of 
cervical screening programs for detection of women at risk for disease progression.

\section{HPV screening methods}

An HPV infection is diagnosed mainly using molecular biological methods because culturing and in vitro propagation of viruses are impractical and serological methods are insufficiently sensitive [26]. These molecular methods include DNA-based assays (e.g., PCR, in situ hybridization, reverse line plot, and the Hybrid Capture 2 [HC2] DNA assay) and RNAbased assays (e.g., mRNA gene expression and protein assays). The DNA-based assays are best for HPV detection and typing in the early stages of infection, but once the disease progresses, RNA-based assays are considered more accurate. However, evidence also indicates that compared with HPV DNA-based assays, both direct and indirect detection of viral gene transcripts may constitute a more specific approach for delineating clinically significant infections. Certainly, HPV oncogene expression and evidence of its deregulation can be monitored through direct detection of viral mRNA transcripts [27]. Figure 1 proposes the most accurate assays (DNA vs. RNA assays) for each stage of disease, and these assays are applicable for use with all HPV types. The use of this proposed approach has already established that HPV-16 and HPV-18 account for over $70 \%$ of all invasive cervical cancers $[28,29]$.

\section{HPV prevalence in the general population in Saudi Arabia}

Despite tremendous efforts, information on HPV prevalence in developing countries generally remains insufficient, and data on the HPV burden in Saudi Arabia are not yet available. However, in Western Asia, the region to which Saudi Arabia belongs, about 2.2\% of the women in the general population are estimated to harbor cervical HPV infection at any given time, and in Asia about $68.5 \%$ of invasive cervical cancers are attributed to HPV-16 or HPV-18 [3]. The incidence of HPV infection in Saudi Arabia is controversial; some researchers claim that HPV infection in Saudi Arabia has the lowest rate in the world (1.9 cases/100,000 women), whereas others claim that the rate of HPV infection among Saudi women is high. Among those studies claiming high prevalence of HPV in Saudi Arabia there is a recent one that described screening of 519 cervical specimens using PCR and a reverse blot hybridization assay. Authors found that 164 (31.6\%) of the specimens were positive for HPV. Among those 164 HPV-positive specimens, 130 showed normal cytology, while 32 showed atypical squamous cells of undetermined significance [30]. A recent observational, epidemiological cross-sectional study conducted between April 2010 and December 2011 at three hospitals in Saudi Arabia investigated the prevalence of HPV in women aged $\geq 15$ years who were attending routine gynecological examinations. The HPV test was conducted using polymerase chain reaction and typed using the SPF10 DEIA/LiPA25 system. Their results showed that in a group of 417 women, HPV-DNA was detected in $9.8 \%$ women. The most prevalent HR-HPV types were HPV-68/73, HPV-18, and HPV-16, while the most common LR-HPV types were HPV-6, HPV42, HPV-53, and HPV-54 [31].

Another study described a relatively high prevalence of HPV-16 or HPV-18 cervical infections among women attending a family medicine clinic in Riyadh (capital city of Saudi Arabia) [32]. The HPV16/18 DNA detection with PCR followed by Southern blotting was performed on 120 women in Riyadh (Saudi and other Arab nationals) during their routine gynecological examinations. Overall, the HPV-16/18 prevalence was $31.6 \%$. The prevalence of HPV-16 alone was $13.3 \%$ and HPV-16 as a mixed infection with HPV-18 was $15 \%$, totaling $28.3 \%$ for all HPV-16 infections. The prevalence of HPV-18 alone was 3.3\%, hence $18.3 \%$ for all HPV-18 infections [32]. A study from the western region of Saudi Arabia examined 40

Figure 1. The development of cervical cancer and proposed approach for DNA and RNA testing. Figure adapted from Jeantet et al., 2009 [29]
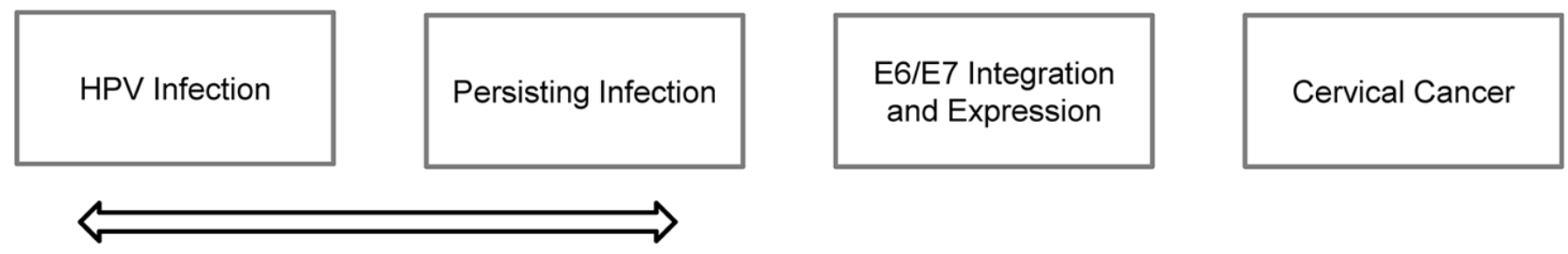

HPV DNA-Based Techniques

HPV mRNA-Based Techniques 
cervical specimens using PCR and BioFilmChip microarray hybridization and reported positive HPV infections in the cervical specimens of $43 \%$ of the women, indicating an alarming rate of HPV infection in Saudi Arabia [33]. The most dominant type observed was HPV-16 (30\%), followed by HPV-18 (8\%), and HPV-45 (5\%).

In contrast to these studies reporting a high incidence of HPV, other studies conducted in Saudi Arabia reported low HPV prevalence. For example, Bondagji et al. examined 485 Saudi women attending a gynecology clinic in the western region of Saudi Arabia using the HC2 high-risk HPV DNA testing technique. They reported that the prevalence of HPV in their clinic was 5.6\% [34]. Another study was conducted using the HC2 method on cervical biopsies from 45 Saudi women. Their results revealed the prevalence of HPV in cervical dysplasia and invasive carcinoma in the biopsy specimens of this Saudi population. The specimens tested positive for HPV in $29.4 \%$ of the patients with cervical dysplasia and $61 \%$ of the patients with invasive carcinoma [35].

The discrepancy in the above reported results may be attributable to the different detection techniques used in the studies. Those studies reporting high rates of HPV used amplification techniques (e.g., PCR and nested PCR), whereas the studies reporting a low prevalence used hybridization techniques (e.g., in situ hybridization and $\mathrm{HC}-2$ ). The hybridization techniques are known to have lower detection limits because they detect the exact amount of DNA present in the specimen. By contrast, the amplification techniques amplify a minute amount of DNA to millions of copies for detection.

\section{HPV prevalence among cervical cancer patients in Saudi Arabia}

Consistent with the incidence rate of HPV among cancer patients worldwide, Al-Badawi et al. screened cervical cancer specimens from Saudi patients and reported a rate of HPV infection of $95.5 \%$ [36]. The most common genotype was HPV-16 (63.4\%), followed by HPV-18 (11.1\%), HPV-45 (4.5\%), HPV33 (3.3\%), and HPV-52, 53, 58, 59, and 66 with 2.2\% prevalence rates. Another study that screened cervical cancer specimens in Saudi women reported that $89 \%$ of the specimens were HPV positive [37]. Eleven different HPV genotypes were detected, including the high-risk HPV-16, 18, 31, 39, 45, 51, 59, and 73 and the low-risk HPV-6, 64, and 70. Consistent with previous studies, HPV-16 and HPV-18 were the most common genotypes.

\section{Other HPV studies in Saudi Arabia}

Advanced studies from Saudi Arabia have also investigated genetic polymorphisms in tumor suppressor genes that may be associated with HPV infection and disease progression to cervical carcinoma. Alsbeih et al. found an association between the single nucleotide polymorphism XRCC1 and cervical cancer in 100 screened cervical cancer specimens, suggesting a genetic predisposition that can be utilized as a biomarker of susceptibility to cervical cancer [38]. Another study, conducted in the Qassim region of Saudi Arabia, screened 40 uterine cervical carcinoma specimens, investigating the expression of HPV-16/18E6 oncogenes and the cell cycle marker P16 (INK4a) as prognostic markers for cervical carcinomas [39]. Their immunohistochemical analysis revealed that $52.5 \%$ of the specimens showed HPV-16/18-E6 positivity and significant correlations between HPV-16/18-E6 expression in cervical carcinoma and nationality, smoking status, and tumor size. The expression of the cell cycle marker P16 was also significantly correlated with high grade stage of cervical carcinoma and HPV16/18-E6 expression. Thus, their results suggested the use of P16 and HPV-16/18-E6 as specific markers for cervical carcinoma.

\section{HPV awareness in Saudi Arabia}

Although cervical cancer is preventable and curable, most women in developing countries, including Saudi Arabia, present at the clinic with advanced stages that require extensive treatment, such as surgery, radiotherapy, and chemotherapy, lowering rates of survival [40]. Therefore, awareness and health education is crucial, especially for educating women about the importance of regular examinations that include Pap smear tests. Unfortunately, many studies have shown a lack of awareness in the Saudi population in general as well as in Saudi healthcare professionals regarding HPV infection, screening, and vaccination [41]. For example, one cross-sectional study used selfadministered questionnaires sent to school teachers, relatives, and friends to recruit 1,023 women from the western region of Saudi Arabia [42]. They found that $37 \%$ of the women who participated had never heard of a Pap smear test, 95\% did not perform Pap smear tests regularly, and most have never had a Pap smear. The majority of the participants $(84.6 \%)$ did not know what HPV was. A recent observational epidemiological cross-sectional study was conducted between April 2010 and December 2011 at three hospitals in Saudi Arabia to document the awareness of HPV infection 
and health-related behavior among Saudi and nonSaudi women attending routine examinations. The results showed that only $32.2 \%(101 / 314)$ of the women were aware of HPV and 89.9\% (285/317) showed an interest in HPV vaccination [41].

\section{Conclusions}

All studies presented in this review reporting on the prevalence of HPV in Saudi Arabia were clinical- or hospital-based. Thus, the true prevalence of HPV in Saudi Arabia cannot be estimated until a national study is conducted covering all 13 provinces of the country and targeting only Saudi women. In addition, awareness programs are needed to educate the public about the importance of Pap smear testing and available vaccinations for the prevention of cervical cancer. And finally, the development of strategic planning to increase the vaccination practice is needed.

\section{Acknowledgements}

We would like to thank King Abdulaziz City for Science and Technology for supporting our research (13-MED2127-20).

\section{References}

1. World Health Organization, (2014) World Cancer Report, Chapter 5.12. Regional Office for South-East Asia. 630 p.

2. CSR, Cancer Incidence and Survival Report: Saudi Arabia (2007). Saudi Cancer Registry. Riaydh, Saudi Arabia.

3. World Health Organization and Institute Català d'Oncologia (ICO) (2009). WHO/ICO Information Centre on HPV and Cervical Cancer. Available at: http://www.who.int/hpvcentre/en/ Last accessed: 22 December, 2014

4. Doorbar J, Quint W, Banks L, Bravo IG, Stoler M, Broker TR, Stanley MA (2012) The biology and life-cycle of human papillomaviruses. Vaccine 30 Suppl 5: F55-F70.

5. Bernard HU, Burk RD, Chen Z, van Doorslaer K, zur Hausen $\mathrm{H}$, de Villiers EM (2010) Classification of papillomaviruses (PVs) based on 189 PV types and proposal of taxonomic amendments. Virology 401: 70-79.

6. de Villiers EM (2013) Crossroads in the classification of papillomaviruses. Virology 445: 2-10.

7. Stanley MA, Pett MR, Coleman N (2007) HPV: from infection to cancer. Biochem Soc Trans 35: 1456-1460.

8. International Agency for Research on Cancer (2012) Biological agents, volume 100B. A review of human carcinogens. IARC Monogr Eval Carcinog Risks Hum 100: 1441.

9. Bernard HU, Burk RD, Chen Z, van Doorslaer K, Hausen Hz, de Villiers EM (2010) Classification of papillomaviruses (PVs) based on $189 \mathrm{PV}$ types and proposal of taxonomic amendments. Virology 401: 70-79.

10. Jean Anderson EL, Sanghvi H, Kibwana S, Lu A (2012) Cervical cancer screening and prevention for HIV-infected women in the developing world. Georgakilas A, editor. Cancer prevention-from mechanisms to translational benefits. InTech: 231-260. doi: 10.5772/2253
11. Raju K (2015) Virus and cervical cancer: role and implication: a review. Biomedical Research and Therapy 2: 220-230. doi: 10.7603/s40730-015-0007-z

12. Münger K, Baldwin A, Edwards KM, Hayakawa H, Nguyen CL, Owens M, Grace M, Huh K (2004) Mechanisms of human papillomavirus-induced oncogenesis. J Virol 78: 1145111460.

13. Zheng ZM, Baker CC (2006) Papillomavirus genome structure, expression, and post-transcriptional regulation. Front Biosci 11: 2286-2302.

14. Grulich AE, Jin F, Conway EL, Stein AN, Hocking J (2010) Cancers attributable to human papillomavirus infection. Sex Health 7: 244-252.

15. Fernandes JV, Araújo JMG, Fernandes TAAM (2013) Biology and natural history of human papillomavirus infection. J Clin Trials 5: 1-12.

16. Calleja-Macias IE, Kalantari M, Allan B, Williamson AL, Chung LP, Collins RJ, Zuna RE, Dunn ST, Ortiz-Lopez R, Barrera-Saldaña HA, Cubie HA, Cuschieri K, Villa LL, Bernard HU (2005) Papillomavirus subtypes are natural and old taxa: phylogeny of human papillomavirus types 44 and 55 and 68a and -b. J Virol 79: 6565-6569.

17. Schmitt M, Depuydt C, Benoy I, Bogers J, Antoine J, Arbyn M, Pawlita M; VALGENT Study Group (2013) Prevalence and viral load of 51 genital human papillomavirus types and three subtypes. Int J Cancer 132: 2395-2403.

18. Chan PK, Picconi MA, Cheung TH, Giovannelli L, Park JS (2012) Laboratory and clinical aspects of human papillomavirus testing. Crit Rev Clin Lab Sci 49: 117-136.

19. Ghittoni R, Accardi R, Hasan U, Gheit T, Sylla B, Tommasino M (2010) The biological properties of E6 and E7 oncoproteins from human papillomaviruses. Virus Genes 40: 1-13.

20. Gnanamony M, Peedicayil A, Subhashini J, Ram TS, Rajasekar A, Gravitt P, Abraham P (2010) Detection and quantitation of HPV 16 and 18 in plasma of Indian women with cervical cancer. Gynecol Oncol 116: 447-451.

21. Narisawa-Saito M, Kiyono T (2007) Basic mechanisms of high-risk human papillomavirus-induced carcinogenesis: roles of E6 and E7 proteins. Cancer Sci 98: 1505-1511.

22. Walboomers JM, Jacobs MV, Manos MM, Bosch FX, Kummer JA, Shah KV, Snijders PJ, Peto J, Meijer CJ, Muñoz N (1999) Human papillomavirus is a necessary cause of invasive cervical cancer worldwide. J Pathol 189: 12-19.

23. Steenbergen RD, de Wilde J, Wilting SM, Brink AA, Snijders PJ, Meijer CJ (2005) HPV-mediated transformation of the anogenital tract. J Clin Virol 32 Suppl 1: S25-33.

24. Apgar BS, Zoschnick L, Wright TC Jr (2003) The 2001 Bethesda System terminology. Am Fam Physician 68: 19921998.

25. Cuschieri KS, Beattie G, Hassan S, Robertson K, Cubie H (2005) Assessment of human papillomavirus mRNA detection over time in cervical specimens collected in liquid based cytology medium. J Virol Methods124: 211-215.

26. Molijn A, Kleter B, Quint W, van Doorn LJ (2005) Molecular diagnosis of human papillomavirus (HPV) infections. J Clin Virol 32 Suppl 1: S43-S51.

27. Cuschieri K, Wentzensen N (2008) Human papillomavirus mRNA and p16 detection as biomarkers for the improved diagnosis of cervical neoplasia. Cancer Epidemiol Biomarkers Prev 17: 2536-2545.

28. Smith JS, Lindsay L, Hoots B, Keys J, Franceschi S, Winer R, Clifford GM (2007) Human papillomavirus type distribution in 
invasive cervical cancer and high-grade cervical lesions: a meta-analysis update. Int J Cancer 121: 621-632.

29. Jeantet D, Schwarzmann F, Tromp J, Melchers WJ, van der Wurff AA, Oosterlaken T, Jacobs M, Troesch A (2009) NucliSENS EasyQ HPV v1 test-Testing for oncogenic activity of human papillomaviruses. J Clin Virol 45 Suppl 1: S29-37.

30. Al-Ahdal MN, Al-Arnous WK, Bohol MF, Abuzaid SM, Shoukri MM, Elrady KS, Firdous N, Aliyan R, Taseer R, AlHazzani AA, Al-Qahtani AA (2014) Human papillomaviruses in cervical specimens of women residing in Riyadh, Saudi Arabia: a hospital-based study. J Infect Dev Ctries 8: 320-325. doi:10.3855/jidc. 4220 .

31. AlObaid A, Al-Badawi I, Al-Kadri H, Gopala K, Kandeil W, Quint W, Al-Aker M, DeAntonio R (2014) Human papillomavirus prevalence and type distribution among women attending routine gynecological examinations in Saudi Arabia. BMC Infect Dis, 14: p. 643.

32. Al-Muammar T, Al-Ahdal MN, Hassan A, Kessie G, Dela Cruz DM, Mohamed GE (2007) Human papilloma virus-16/18 cervical infection among women attending a family medical clinic in Riyadh. Ann Saudi Med 27: 1-5.

33. Turki R, Sait K, Anfinan N, Sohrab SS, Abuzenadah AM (2013) Prevalence of human papillomavirus in women from Saudi Arabia. Asian Pac J Cancer Prev 14: 3177-3181.

34. Bondagji NS, Gazzaz FS, Sait K, Abdullah L (2013) Prevalence of high-risk human papillomavirus infections in healthy Saudi women attending gynecologic clinics in the western region of Saudi Arabia. Ann Saudi Med 33: 13-17.

35. Sait KH, Gazzaz FS (2011) Molecular tests to detect human papillomavirus infection in patients with cervical dysplasia and invasive cervical cancer in Saudi Arabia. Pathol Lab Med Int 3: 25-29.

36. Al-Badawi IA, Al-Suwaine A, Al-Aker M, Asaad L, Alaidan A, Tulbah A, Fe Bohol M, Munkarah AR (2011) Detection and genotyping of human papilloma virus in cervical cancer specimens from Saudi patients. Int J Gynecol Cancer 21: $907-$ 910.
37. Alsbeih G, Ahmed R, Al-Harbi N, Venturina LA, Tulbah A, Balaraj K (2011) Prevalence and genotypes' distribution of human papillomavirus in invasive cervical cancer in Saudi Arabia. Gynecol Oncol 121: 522-526.

38. Alsbeih G, Al-Harbi N, El-Sebaie M, Al-Badawi I (2013) HPV prevalence and genetic predisposition to cervical cancer in Saudi Arabia. Infec Agent Cancer 8: 15-22.

39. Omran OM, AlSheeha M (2014) Human papilloma virus early proteins E6 (HPV16/18-E6) and the cell cycle marker P16 (INK4a) are useful prognostic markers in uterine cervical carcinomas in Qassim Region-Saudi Arabia. Pathol Oncol Res doi:10.1007/s12253-014-9801-y.

40. Manji M (2000) Cervical cancer screening program in Saudi Arabia: action is overdue. Ann Saudi Med 20: 355-357.

41. Al-Darwish AA, Al-Naim AF, Al-Mulhim KS, Al-Otaibi NK, Morsi MS, Aleem AM (2014) Knowledge about cervical cancer early warning signs and symptoms, risk factors and vaccination among students at a medical school in Al-Ahsa, Kingdom of Saudi Arabia. Asian Pac J Cancer Prev 15: 25292532.

42. Gari A, Asiri A, Mohammed A, Al-Malki F, Melibari M, AlSheikh M, Kalantan M, Al Qethami N, Futtiny S, Al-Essi S, Al Shomrany Y, Alihiby A (2102) The awareness of the HPV's association with cervical cancer and the HPV vaccine among Saudi females. Life Sci J 9: 2538-2546.

\section{Corresponding author}

Mohammed N Al-Ahdal

Department of Infection and Immunity, King Faisal Specialist

Hospital and Research Center MBC 03, P.O. Box 3354 Riyadh

11211, Saudi Arabia

Phone: +966 114427867

Email: ahdal@kfshrc.edu.sa

Conflict of interests: No conflict of interests is declared. 